\title{
PLANNING FOR FUTURE URBAN GROWTH
}

\author{
HENRY FAGIN*
}

INTRODUCTION

I shall deal with two aspects of planning for future urban growth: current changes in the process of planning and the kinds of urban structures likely to result from that planning. In treating the former, I shall describe both traditional and new techniques of planning, stressing key characteristics of the new techniques that are dramatically modifying present planning processes. With regard to the substantive aspect of future urban growth, a vast and difficult subject, I shall not attempt so much to sketch a comprehensive picture of urban life in the next century as to indicate the substantive modifications that will tend to flow from the changes in the planning process. Finally, in the concluding section I shall suggest what might be done to expedite the development of planning processes better adapted to meeting the needs of contemporary urban life.

We plan within a particular context, and the context validates the particular methods we use. The context of our urban planning has been changing notably throughout this century. Though many facets of modern life date from the Industrial Revolution, changes that accelerated drastically after World War II have plunged us into a new urban epoch-an epoch characterized by greatly increased scales of urban size, complexity, and interaction.

The pre-existing mechanisms for handling urban problems have been strained to the breaking point. In addition to being overwhelmed by the sheer magnitude of urban development, we have been baffled by its complexities, unable to see clearly just what has been occurring and in what ways or to unravel the mysteries of cause and effect. And we have been frustrated in our attempts to grasp the urban scene in its dynamic aspect as essentially a cooperative process with unprecedented levels of interaction and interdependence.

Thus, we have found it difficult to pin down the context of our planning-the more so because the whole has been changing so rapidly before our very eyes. What clearly does emerge, however, even from our limited perception is a sense of urgency about the need to improve our planning processes precisely so as to gain the capability of handling adequately the three unmistakable urban characteristics just cited: great size, great complexity, and great interaction.

One ubiquitous response on the part of urban governments to the new problems inherent in the contemporary urban context has been a marked increase in the number and variety of governmental activities. This has been so not only in terms

- B.Arch. 1937, M.S. in Planning and Housing, I938, Columbia University. Professor of Planning, Department of Urban and Regional Planning, University of Wisconsin. 
of an intensified regulation of private development, but also in terms of the direct provision of public facilities and services. Initially, the new programs tended to be logical extensions of existing ones, and they were planned and administered readily enough within the framework of existing departmental responsibilities. Characteristically, however, certain of the more recent programs have grown out of perceptions of problems that arose from fresh ways of looking at urban events. Indeed, for this reason, the new frames of reference tend, more often than not, to cut across present departmental jurisdictions. Attention today focuses on poverty, jobs, aging, urban renewal, area development-problems that cannot be delegated neatly to the traditional governmental departments but rather must be tackled cooperatively by all of them in shifting combinations.

Another characteristic governmental response has been a new level of involvement in the field activities by which urban areas are built and rebuilt-developmental processes formerly almost entirely left to the private sector of the economy. In urban renewal, in industrial development, and in aspects of new community design and construction, municipalities and their agencies plan, negotiate, buy and sell, manage, and service in ways that contrast sharply with the very limited proprietary roles that were undertaken even in the days of the New Deal.

Moreover, this direct participation in developmental activities has itself brought urban governments sharply up against an array of social and economic problems that previously were largely left to the concern of private or voluntary groups. In fact, some of the attention to broad social problems noted above grew directly out of governmental experience in the development field. The need to clear land in particular places, for example, led inexorably to involvement by urban governments in race relations, alcoholism, and improved concepts of public housing. In the simpler circumstances of the past, the sharp distinctions separating social, economic, and physical development issues in legislation and administration (and in planning) were tolerable. But today the lines are increasingly violated as municipalities become involved in developmental actions and experience vividly the seamless web that is the essence of the urban condition.

This experience is generating two seemingly divergent responses. On the one hand there is some tendency for municipalities to centralize responsibility for the "developmental" activities in a new kind of agency that is not clearly either "line" or "staff" in the traditional sense, but that plans comprehensively, coordinates, and acts simultaneously. Philadelphia's Development Coordinator, for example, is a sort of line operator within the staff office of the city's Managing Director. In some cities the whole cluster of public housing, renewal, inspection and code enforcement, construction expediting, and city planning has become the responsibility of a single new department. The inclusion of the function of planning for the physical development of the municipality alongside various entrepreneurial activities is worthy of special note. 
On the other hand, a different logic rules in other places where the integration of programs scattered across departmental lines is sought through the creation of more powerful methods of influence and greater comprehensiveness in the scope of the overall planning units. Pursuant to this approach, after recent reorganizations one is beginning to find fiscal planning, budgeting, overall program initiation, and planning for physical development all collected close to each other and to the chief executive.

Each of these approaches has its corollary. Planning in the unified development agency tends to become action-oriented, specific, detailed, and concerned primarily with those geographic areas within the community where physical change is underway or imminent. In contrast, planning in the integrated overall policy agency tends to confront a wider range of problems with which, given their mass and complexity, the centrally situated planning agency tends to deal on a more generalized basis. It stresses the interactions, the mutual impacts, the opportunities for coordination and cooperation. It furnishes a unified common framework in terms of an integrated set of overall goals and policies, a single guiding plan for the whole spatial environment, and a comprehensive budget for allocating total resources among all contending needs for services and for facilities.

In either case, the close association of various strands of public planning that traditionally have been conducted in a degree of isolation from each other, is having important effects on all of them. The land use planners are under pressure to deal more extensively and more precisely with the social and economic inputs to their work as well as with the potential impacts of physical structures on the other facets of life. The city planners in turn are challenging budgeting and programing planners to expand their attention in two directions: first, to make current decisions after assaying bolder projection of considerations into the future; second, to take more account of the total developmental process, private and public, when setting governmental policy.

Another significant aspect of today's urban context for planning is the way the flow of development has spread across old political boundaries and created a multitude of new ones. For lack of areawide operating agencies, metropolitan leaders have attempted to achieve at least a modicum of coordination and cooperation by establishing regional planning agencies. To be sure, the solution of regionwide problems requires more than mere agreement on plans. Certain types of facilities and services are essential and must be provided if satisfactory urban conditions are to be achieved. And certain kinds of adjustments must be made in local programs of action and regulation.

Given the urgency of the problems and the lag in the creation of effective metropolitan governmental structures, much of the burden of financing metropolitan improvements has fallen on the federal government-and partly on the states as well. Federal experience with grants to the localities in a number of different program 
fields has proved the need for more effective administration at the local level of programs financed by federal funds. In response to this experience, the Congress has increasingly moved toward promoting improved regionwide administration in the nation's urban areas. This federal pressure toward the better handling of regionwide problems through the establishment of metropolitan agencies of various kinds is itself a very important new factor in the context of the urban environment.

Finally among these major new elements of the situation, there is a somewhat technical concept that is rapidly coming to influence the fundamental way in which we approach urban planning. This is what is loosely called the systems concept. It is an application of a set of ideas that has also tremendously affected management methods in the private sector of the economy. The idea of interacting systems has long been familiar to us in terms of human biology, as when we deal with the alimentary, nervous, skeletal, or circulatory systems and their interrelationships. What is new is not the general idea, but the rigor with which the systems approach is applied and the proliferating use of new computation methods to aid the solution of systems problems. As we shall see, the general emphasis on this approach that has been in the air in recent years has begun to modify traditional methods of planning as well as ways of organizing the governmental programs that flow from the planning process.

\section{The Traditional Planning Process}

The traditional application of systematic, long-range, comprehensive planning to prepare for urban change has focused primarily on the physical aspects of the metropolis. Community planning has been land use planning. Its recommendations have been mainly things that might be shown on a map or seen from an airplane flying over an area at a height, say, of one thousand feet. It has dealt with the densities and general arrangements of structures used for various categories of purposes, with the public facilities needed in connection with the buildings, and with the patterns of streets and other transport lines that provide circulation throughout the community. While focused on the spatial aspects of the physical environment, urban planning has traditionally paid due regard to nonphysical (social and economic) factors of two kinds: those that act strongly on the physical environment and those that are acted upon strongly by the way the physical environment is arranged.

This framework, broad in some ways but nevertheless definitely restricted, appears to have been in part self-imposed and in part imposed from the outside on the planning movement. It was self-imposed to the extent that the movement came to be dominated by people most comfortable when dealing with spatial design-men initially drawn from the ranks of the design professions of architecture, landscape architecture, and civil engineering, and later turned out in a similar mold by planning schools that were nurtured by one or another of the three. Though there were public 
of land significant to its potential for community expansion as topography, soil types, vegetation, sub-surface conditions, patterns of land values and property ownership. Implementation studies looked into the municipal debt structure with an eye to capital expenditure recommendations, examined existing regulatory instruments-the zoning ordinance, building code, subdivision regulation, official map-to assess their adequacy and to recommend improvements.

On completion of these surveys - and others that might be considered appropriate in particular places-the so-called planning phase was begun. First came the land demand studies. Projections were assembled from all the surveys and combined to form a consistent set of curves representing estimates, among others, of persons, households, employment, houses, business establishments, and vehicles. These projections were then converted into the common measure, acres of land required for each type of land use. Based on the adequacy of lands in present use, estimates were then made of the total quantities of land that would have to be developed to replace uses displaced by clearance and to accommodate the expected increments of land use. Design studies followed. Their purpose was to develop a concept of community form-a logic to guide the spreading out on the land of the land-use increments already estimated. This logical form, frequently named the master plan, was the instrument by which the planners coordinated interacting land uses and related land uses to the public facilities needed to serve them. The quality of the plan was to be measured in terms of the beauty of the resulting development, the convenience experienced by the citizens, the efficient operation of enterprises, public and private, and the capability of the community form to accommodate growth without disruption or congestion.

Studies about implementing the plan followed. These produced recommendations to improve various control documents and their administration-for example, land development regulations, occupancy codes, urban renewal programs, and capital project programs and budgets.

Finally, it has been typical of urban planning studies, especially in dynamic urban regions, that forces beyond local control and trends beyond local anticipation caused relatively rapid obsolescence both of the plans resulting from the planning process and of their implementing devices as well. Within a decade there was likely to be a demand for a new series of studies and a new community plan. Then the whole cycle started anew and after several years of work a revised plan supplanted the old one.

II

The Emerging New Planning Process

Before describing certain significant ways in which the planning process just outlined is changing, let me hasten to say that a great deal of what was done did influ- 
administrators, lawyers, and social reformers of various backgrounds in the early ranks of the planners before World War I, these men either accepted the emphasis on the physical environment natural to the design-oriented planners or they turned toward other pursuits. The issue was fought and settled before I920, and the city planners found their chosen field quite lucrative in the building boom that followed.

This separation of planning for the physical environment from planning for needed programs in the social and economic realms was challenged briefly again within the National Resources Planning Board in the late r930s. By that time, however, the distinction was so firmly established that it was deemed politically dangerous to modify it, given the Congress' apparent willingness to support planning for better local physical patterns but its apparent dislike for the systematic planning of programs directed toward improving social or economic welfare. Before long, Congress terminated the whole NRPB program. It is ironic that, despite this retreat, many of the New Deal's social and economic programs not only survived but have grown in importance ever since.

At the municipal level, however, city planning, with its focus on land use and circulation patterns, has indeed flourished. Influenced first by the planning schools and the successful planning practitioners, and reinforced by legislative provisions written in the same spirit, a uniform approach to planning gradually evolved. It took the form of a standardized series of studies that became the basis for the preparation of plan reports and around which planning curricula tended to be organized.

A city planning effort typically began with a survey of existing conditions and trends and a projection of all numbers involved into the twenty-year future. Land use studies mapped and measured the acreages devoted to each of about ten categories of land use-one-family, two-family, and multi-family residential; commercial; industrial; park; public; and so on. Economic base studies traced employment changes in the community's industries and suggested measures that ought to be taken to strengthen the local economy. Population studies examined what had been happening to birth, death, migration, family formation, and labor participation rates as well as the prospective changes in the composition of the community's population. Housing studies evaluated the quality and counted the quantity of the community's housing stock, pinpointed areas of blight, and recommended measures to improve residential neighborhoods by the removal of substandard structures and the provision of additional community facilities. Circulation studies mapped and classified the street system and recommended new or widened streets to correct places of congestion and extensions to serve areas still undeveloped. Community facilities studies inventoried schools, playgrounds, parks, firehouses, police stations, and so on and mapped their respective service areas in relation to present and prospective population distributions. Utilities studies dealt with the capacity and locations of lines for carrying water, sewage, storm drainage, gas, electricity and power. Land development studies examined and mapped such physiographic and economic characteristics 
ence the development of livable, well-functioning communities. Particularly in situations where social and economic conditions were healthy, the focus on producing excellence in the physical environment proved rewarding, both to the community and to the professional planners involved. The well-to-do suburban town with a steady but not excessive influx of successful city families, favored by expanding retail and service market areas, was the prototype scene for successful planning. The shortcomings of the physical emphasis were relatively unimportant wherever the local problems remained predominantly in the spatial realm. The real challenge, successfully met in few places, was how to plan effectively where deep-seated political conflict was generated by difficult social problems and where strains threatened the economic viability of the community. In such places, the social, economic, political, and spatial aspects of the community are so extensively interwoven that conclusions reached within the restricted horizon of any one of these several frameworks are bound to prove one-sided, warped-color-blind, so to speak.

Whatever the fate of the old theoretical debate over the proper scope of urban planning, the inescapable events of the rapidly urbanizing world have been producing an unmistakable transformation not only in scope, but in organization, method, and program as well. Of great significance has been the evolution of more thorough public budgeting, already alluded to above, as an approach to the coordination of urban activities parallel to master planning. We have seen that two major facets of traditional city planning have been influencing the scope of public budget studies: one, the value of medium- and long-term projections of revenues and expenditures; and the other, the value of considering public facilities and services more extensively in direct relationship with the changing characteristics of the community as a whole, taking a positive and active role with regard to the private sector as well as the public. The development of performance and program budgeting has been an important advance toward a capacity to deal adequately with the public finance aspects of urban economic growth and change.

Pressure for more effective urban planning comes not only from the inside, but also from state governments and especially from the federal government. Faced with huge outlays to help remedy unbearable urban ills, the larger governments are requiring-and helping to finance-substantially more thorough local planning programs. From their larger vantage point they are pressing for two kinds of extension of urban planning: first, toward region-wide planning, in recognition of multimunicipal needs for services and of the mutual impacts of community programs across municipal boundaries; and second, toward the unification of planning, aimed at concentrating federally- or state-supported programs in a coordinated attack on urban problems. An important drive appears to be underway to align the separate programs in the fields of poverty, the aging, the youth, area development, recreation, housing, highway construction, mass transportation, employment, and education. 


\section{III}

\section{The Systems Approach}

Perhaps the level of coordinative planning implied by the foregoing awareness of urban interconnection would have been an impossibility a decade or two ago. It should be remembered that traditional city planning recognized the importance of coordinating a number of interacting systems. The master plan was essentially an instrument for so doing. But one of the pressures on the planner to remain within the realm of the physical environment, to keep to a general level, and to focus primarily on the provision of facilities rather than to deal more broadly with urban services must have been the sheer limitation imposed by existing methodological techniques. The planner measured like an engineer, analyzed like a geographer, and designed like an architect. The drafting board was his stock-intrade. His ability to handle simultaneously several interacting systems was limited by the number of thicknesses of tracing paper he could see through, so to speak. Indeed, the initial use made by planners of the powerful new computational instruments that are revolutionizing the rest of technology was to substitute mathematical for graphic means of perceiving inter-relationships among urban sub-systems. Carroll's influential work to establish the connections between land uses and transportation flows in the metropolis (developed successively in Detroit, Chicago, Pittsburgh, and now New York) exemplifies this thrust. The planner now works with twenty overlays at a time rather than just three or five.

As I have already suggested, the generic term for this new approach is systems planning. This is the basic concept underlying what is variously called operations research, administrative science or, in the planning field, comprehensive metropolitan transportation planning. In terms of urban planning, the systems approach is now identified most closely with transportation planning because the systems concepts were first applied in metropolitan highway studies. The approach, however, is spreading rapidly to the whole field. For example, some of the most interesting applications currently relate to large-scale urban renewal programs. It seems to me unlikely, however, that these efforts will long remain relatively isolated from each other. The whole logic of federal and state pressure toward understanding and handling the repercussions of various programs on each other, toward taking advantage of all possible additive effects, is tending to bring the various urban subsystems into a common framework for analysis and creative action.

Let us now consider in greater specificity what happens to the urban planning process under the disciplining impact of a systems approach. Mainly it forces a much more explicit identification and description of the sub-systems that together constitute the whole environment. I mentioned earlier certain studies that have come to characterize urban planning programs. Some of these do distinguish essential sub- 
systems, but the list is quite incomplete and the entries are not coordinate with each other.

Here is another list, not structured logically in terms of a division of responsibility within the planning office nor in terms of convenient report-writing categories, but structured by the way activities are organized in the real world. These are some of the most important sub-systems of the urban system as a whole:

(I) National and sectional context. This system comprises the larger scheme within which a particular urban region functions. It describes the system of transactions that take place between a region and significant regions elsewhere, and it describes the workings of each larger system.

(2) Physiography. This system is itself a series of sub-systems covering various elements of the natural environment, some of them already cited above in connection with traditional planning. The difference, however, is that the component elements are now identified and described more completely by reference to the overall eco-systems of the region.

(3) Structures. This system comprises all man-made improvements to the environment: buildings, roads, bridges, docks, airports, and so on.

(4) Demographic pattern. This system includes not only the distribution of the population in a geographic sense, but also the composition of the population, the elements that bring about population change, and other facets of the social structure of the region.

(5) Production and distribution pattern. This system describes the characteristics of the region's commercial, industrial, governmental, and other similar establishments.

(6) Consumption patterns. This system comprises households and other consumers. It deals with their geographic distributions as well as their buying patterns.

(7) Land uses. This system, itself composed of many sub-systems, describes the urban activities, social and economic, in their spatial patterns. It includes areas at least for production, storage, exchange, transport, residence, recreation, services, and conservation. It differentiates density patterns as well as degrees of diffusion or of centralization. It distinguishes, for example, among patterns of even urban spread at decreasing densities from a center, patterns with strong nodal articulation, and linear patterns of various sorts.

(8) Communications. This is the set of sub-systems comprising the networks for transporting messages, persons, goods, energy, water, and wastes.

(9) Accessibility. This system also is a function of several other systems taken in combination, particularly land uses and communications. It describes the potential connections among establishments carrying on various interdependent activities via the available communications networks, especially the transportation links. Accessibility may be modified for a particular establishment either 
by a change in the communications networks or by a change in the locations or intensities of other land uses.

(10) Urban interaction. This system describes the actual rather than the potential interchanges or linkages among activities via the communications networks. It embraces informational, commodity, financial, and other communications flows. It includes as a sub-system, for example, the traffic patterns of the region.

(II) Urban design. This system expresses the aesthetic pattern that results from the interplay among buildings and other structures and the landscape.

(12) Land and improvement values. Although inherent in a combination of subsystems already identified, this system also has its own inner logic and dynamics. It is expressed through the pattern of assessed values adjusted to reflect market prices.

(I3) Land ownership. This system describes such aspects as the sizes and locations of holdings, the types of owners, and the types of ownership.

(I4) Government. This is another system that comprises many sub-systems each significant for urban planning. It includes jurisdictions, revenue and expenditure patterns, funancial capacities, governmental services, and sub-systems of regulation such as development codes, and so on. It should be noted that this general category could be expanded to distinguish among all the separate systems into which particular governmental programs are subdivided.

The mere recital of these fourteen important urban sub-systems offers some indication of the dimensions of the problem of understanding each important sub-system as well as their major interrelationships. Any one of them by itself is very complex. Yet if we were to continue to pay close attention to some while virtually neglecting others, we would continue to create or exacerbate grave problems that go unnoticed in one sphere while one concentrates on problems perceived in the next. Fortunately, certain elements of a systems approach promise to render the overall problem reasonably manageable.

First, there is the concept that degree of loss through sub-optimization can be controlled. That is to say, we can continue to operate recognizing in a practical sense that many, if not most, decisions can be made adequately within the framework of less than a total view. What is needed, however, is a systematic way of identifying the resulting negative impacts at least to the extent that they threaten to become substantial and to remedy those that do prove potentially serious. This suggests the need for both decentralization and centralization-for a process that relegates decisions as far downward as feasible, but with a checking mechanism that eventually brings programs into juxtaposition and adjustment before they reach the stage of action. Such an approach now appears attainable.

The second element helping toward manageability is the development of new methods of large-scale data management. While our elastic concept of relevance is 
rapidly increasing the amount of information we should like to have available to formulate and weigh urban development policy, the capacity of modern computer installations is expanding at an even faster rate. If, as is to be expected, the third and fourth generations of computer equipment that will come into use in the next decade prove as much advanced over what we now use as the second generation was over the first, then one serious present restraint on data development will have been removed. We will be able to study whole urban metropolises limited rather by our own intellectual insufficiencies (which will then prove crucial) than by mechanical problems, which now occupy much of our attention.

A third important aspect of a systems approach is its insistence on a precise formulation of the objectives to be sought in urban planning. It is true that some planners have always listed what they called community goals-sometimes even after consulting the community. Given the extremely loose connection between the statement of general goals and their specific expression in the master plan, however, there was little incentive to think clearly and comprehensively about the goals. There was no disciplining mechanism that exposed inconsistencies or conflicts among seemingly acceptable goals, nor any way of measuring the extent to which the proposed plans actually would affect the goals.

In this regard the systems approach enforces a rigorous handling of goals. It demands specificity and detail in the statement of objectives. This renders them communicable, debatable, and above all useful. One of the most interesting studies in the urban planning field is currently being conducted within the Twin Cities metropolitan planning program. There, an exhaustive examination is being made of the community's general scale of values, the relation of these to environmental goals, and the formulation of goals in objective terms capable of measurement and translation into design specifications and decision criteria.

The fourth simplifying element of the systems approach is the vital and central concept of the mathematical model. This is, as is fast becoming well-known, the idea of formulating problems in such a way as to represent complex real situations by simplified imaginary constructs, so fashioned as to yield workable approximations of the operations of the real world while remaining amenable to mathematical analysis and manipulation. Such models, used now in almost every field of science or social science, make it possible to handle simultaneously very large numbers of considerations. They make it feasible to analyze not merely individual proposals, but the combined potential effects of numerous combinations of elements. In terms of urban structures, they open up the possibility of moving beyond the consideration of physical facilities in their inert state, which has characterized so much planning in the past, to an involvement also with facilities in action-or services and levels of service. This capacity then opens the technological door to close collaboration between financial and physically-oriented planners. 
Fifth, the systems approach promises a substantially higher level of accuracy in the prediction of the potential impacts of things proposed. It is worth noting here that informed people, drawing on their intuitive powers, have sometimes in the past been very successful and accurate in explaining what would happen if their advice were taken. To depend mainly on individual brilliance for this vital part of the planning process, however, would be to neglect the advantage that might be taken if what I call "social progress in predictive capability." The systems approach, by making transparent the assumptions, parameters, and other elements of a prediction of cause and effect, makes it possible to discover what parts of the predictive process have proven to be valid and what parts warrant further work.

And sixth, the development of mathematical models as part of the systems approach makes it possible to deal much more extensively and authentically with change over time than was the case when graphic methods dominated urban planning. Some ten years ago in another paper printed in this Journal, ${ }^{1}$ I speculated about the desirability of regulating the timing and flow of urban development as well as its eventual pattern. Methods for doing so had then not yet been developed. More to the point, one's grasp of future sequences was then very limited. Happily, few communities rushed to take my advice. In the intervening years, however, we have indeed developed a capacity to program public measures in dynamic relation to the changing external environment. Our analytic tools now enable us to visualize urban change and the consequent changing service demands that are likely to unfold from time to time in future years.

Taken together, the six factors are bringing about a dramatic change in the education of planners, in the organization of the planning process, in the methods used for planning, and in the levels of responsibility entrusted to professionals in the field. Given the broad spread of the kinds of considerations seen to be relevant, it is not surprising to find interdisciplinary collaboration a hallmark of current professional staff work wherever urban problems are being tackled within a planning framework.

\section{IV}

\section{The Nature of Future Urban Development}

It is to be hoped that the deep-going changes that have been occurring in the planning process will be experienced presently in terms of greatly improved urban environment. It is, of course, much too early for this to have become manifest. Even for a single public improvement, a new medical school building or a highway link, more than a few years must pass before the impact is fully felt-the more so for a system or network of improvements. Indeed, despite the best efforts, it sometimes happens that the elements of a system that have to be initiated first temporarily worsen the total situation. Thus, the validation of a systems approach to the planning

${ }^{2}$ Fagin, Regulating the Timing of Urban Development, 20 LAw \& ContEMr. Pros. 298 (1955). 
of a large urban area is not likely to be established empirically short of several decades.

At present it is the theoretical reasonableness of the approach that appeals to so many planners rather than any impressive record of substantive achievement. For, the approach has two invaluable assets. It enables the planner to confront enough elements of the total situation to feel that he is approximating the real world. And it provides a usable substitute for an empirical testing of conclusions by enabling a computer-assisted simulation of potential results. The systems approach makes it possible to simulate the evolution of the metropolis on a speeded-up basis so that the probable respective impacts of various test alternatives can be traced in advance and their comparative advantages assessed.

Direct evidence is lacking, then, to compare potential future urban development under the influence of new planning technology with what has been resulting from past methods of planning. One can only indicate intuitively some of the likely differences.

An interesting foreshadowing of such differences can be recognized in an historic example. In the Ig2os Clarence Stein and Henry Wright approached the housing problem in the context of the whole community. In designing Radburn, New Jersey, they considered not only the house on its lot, but the lot in relation to all the other establishments used during the day by the members of the family. The resulting design produced an environment dramatically different from any then existing. Indeed, its innovations have not yet been digested fully by the general real estate industry, though its imprint is unmistakable in the best communities being developed today throughout the world.

This example suggests once again that the fundamental essence of the systems approach is not the use of computers to solve the equations of mathematical models but the habit of looking at the whole and the part at the same time, seeing them in dynamic relationship. This has been very much an integral part of the traditional approach to planning from the outset. What is specifically new in the current ferment within the planning movement, however, is the strikingly expanded power to deal successfully with complex systems that is inherent in the new scientific technology of the day. In this latter factor lies the significance of the computer to the field of urban planning.

The potential impacts of our expanded capacity to deal with urban problems of great magnitude, complexity, and interaction will become more tangible, I believe, if I describe them in relation to several crucial aspects of urban growth.

(A) Jane Jacobs and others, though weak in the remedies they prescribe, have accurately exposed the folly of compartmentalizing our thinking about urban renewal. A number of stereotypes that stamped past attitudes toward urban structure are being searchingly challenged and re-examined currently. Take, for example, the 
sharply defined districting of land uses that characterizes communities designed two-dimensionally in terms of simple land-use categories. It seems to me probable that in planning future urban growth and change, we will tend to segregate activities on a much finer scale than at present. There may be a great deal of vertical zoning, so to speak, with different uses stacked in the same building. Skillful design will promote the close integration of diverse things. The urban scene will be enlivened by a striking degree of local variety, far beyond what we find now in most planned areas. The new center of Rotterdam, with its mixture of town houses and apartments and office structures and theaters and shops, seems more indicative of the urban future than the current American generation of municipal master plans, still dominated by the ring or the sector theories of urban land economics. To be sure, such a variegated fabric will require extremely sensitive handling of exact relationships, in both the design and regulation phases of urban construction. Here is an exciting and important new field, for administrative and legal exploration.

(B) The direction of evolution in the nineteenth-century metropolis led toward a concentric separation of home and work place. The linkage between a man's house and his job was sacrificed to shorten the linkages among productive establish. ments as business areas were drained of residences. Tremendous diurnal waves of commutation built up, flooding the railroad and streetcar channels that radiated from the central cores. Around Igoo the biggest East Coast cities were forced into an era of subway and elevated transit construction to keep up with the massive numbers involved.

By mid-century, however, a new and widespread distribution of automobiles among the population, particularly in the suburbs, suggested a fundamentally different urban structure, and many industrialists moved to take advantage of the suggestion. In essence, a locational shift was initiated to shorten the link between home and work place even though this meant increasing the linkages among work places-at least in terms of distance. Coupled with other technologic changes which gave advantages to production on cheap, outlying land, the logistics of automobile commuting began to turn the metropolis inside-out. But the results are by no means convincingly better.

We now are faced with a great debate as to which form is superior. Nobody today can prove the answer, one way or the other. Moreover, still dominated by the conceptual limitations imposed by colored-map thinking, much of the current argument is over paper relationships, not real ones. For example, we have hardly made a beginning in the development of adequate methods of metropolitan benefitcost analysis. My hunch is that quite unexpected structural conceptions about urban form will arise in the next decade, when this systems problem par excellence has been investigated with the powerful new tools of analysis and design we are just learning to use. My only firm conviction about the outcome is that the type of spread city we are still building today will be decisively rejected. 
(C) The anticipated organizational integration of planning for the physical, financial, and programmatic aspects of the urban environment, which I described earlier, will tend to place proposed spending on public works in a more useful perspective. Both the favorable and unfavorable potential impacts of proposed physical arrangements of urban activities upon the qualities and costs of urban services will become much more clearly evident. Within this more rigorous institutional setting, the true and complete costs of alternative patterns will be sought and, to the extent feasible, weighed. It seems to me likely that the resulting decisions will tip the balance extensively in the direction of ( $\mathrm{x}$ ) higher-density living, (2) more intensive use of mass transport, and (3) greater linearity in the spatial organization of urban activities in recognition of the linear essence of most communications media-pipes, power transmission lines, and controlled channels for vehicles. Short of technologic innovations that might eliminate this factor, the reticulated metropolis may become the urban form of the future, though the scale of the network has not yet been investigated systematically.

(D) The division between the private and the governmental sectors of future urban activity is likely to differ significantly from the present mix. The change, however, may not so much involve the overall balance as it will the detailed division of responsibilities. The service contract concept, applied as in Los Angeles County between small and large units of government or as in Philadelphia between the city and private service companies, is likely to blur the classical dichotomies of public versus private and local versus metropolitan. This in turn should free the urban planners from certain historic restraints that have reflected these dichotomies. Transit systems integrating local and express, city and suburb, bus and train, wiil become feasible to bring high quality low-cost service to much larger geographic areas. The design of urban development in relation to the natural landscape will be liberated in considerable measure from the heavy hand of municipal mercantilism. Largeścale regional aesthetics will come into its own. Comprehensive economic and social considerations will play a greater role and local politics a lesser role in the shaping of urban change. The capacity to handle urban growth (including urban renewal in developed areas) with less municipal constraint will reinforce another new factor, this one growing out of technologic and institutional changes in the building industry itself. The scale of the individual urban construction project has increased steadily since World War II. The exception that was Levittown is fast becoming the predominant method of community building. Given the successful handling of the opportunity for close collaboration between public planners and private planners to which I have alluded, the new context of urban developmental activity should enable an early realization of the contemporary concepts that will be coming out of governmental and private planning offices, stimulated by the new planning processes that now lie close at hand. The effects of this new scale and quality of planning will not only be to bring many urban elements now out of control into 
an harmonious relationship with the whole. It also will release from governmental regulation many elements that we have felt compelled to control publicly because of the extreme fragmentation of responsibility that has characterized the urban past. The change will be felt in the quality and economy of urban services. It will be seen vividly, I am convinced, in a new urban beauty that the new urban processes will release.

\section{V}

\section{What Must Be Done}

The new planning processes and the new urban forms $I$ have been discussing will not automatically come about. They will be brought into being by tough and persistent effort.

An essential restructuring of the planning process is resisted by the now-separate professional and governmental institutions that have flourished and prospered under the present frameworks. There must be public pressure on these institutions to adapt to the needs for unification and integration that grow out of the very essence of the metropolitan culture. A greatly encouraging sign of the times is the new Interprofessional Committee on Environmental Design that has been established by five design professions: planners, architects, landscape architects, civil engineers, and industrial designers. A next step toward better integrated urban planning might be the convocation of a parallel committee of urban planners, budget officers, and the emerging corps of comprehensive governmental policy aides.

Twenty years of unrewarding struggle over metropolitan government matched by twenty years of disappointing experience with floating metropolitan planning agencies should warn us to look to some different formulation of the problem of urban coordination. The present mood of the Congress, determined to bring about effective and democratically responsible means for coordinating metropolitan programs that are federally aided, offers a fresh opportunity for invention in the field of urban institutions. Furthermore, recent court decisions have introduced a new fluidity in municipal-county relationships. This, then, would be a fruitful time for a new concentration on the problem of government in the metropolis.

Finally, this is the period of a vast emphasis on the need for education. Surely, a vital part of this is the re-education of the educated. American leaders and the public alike remain seriously uninformed about exciting urban forms that are being innovated in Scotland and Scandinavia and England and Europe-in fact right here in America. The level of what we demand in the quality of the everyday environment must be dramatically raised.

For the urban landscape is an inexorable reflection of the urban culture that creates it. The fundamental problem is to create in the consciousness of our culture 
a vision of what the culture can now create. As the late Henry S. Churchill so eloquently put it: ${ }^{2}$

A city plan is the expression of the collective purpose of the people who live in it, or it is nothing. For in the last analysis, planning is not just yielding to the momentary pressures of fugitive groups, nor is it even the making of beautiful maps encompassing future hopes. It is something far more subtle; something inherent and ineluctable-the unspun web in the body of the spider.

${ }^{2}$ Henky S. Churchill, The City Is the People i 86 (I945). 